\title{
Hybrid super-lattices of graphene and hexagonal boron nitride: Ferromagnetic semiconductor at room temperature
}

\author{
Rita Maji and Joydeep Bhattacharjee \\ School of Physical Sciences \\ National Institute of Science Education and Research \\ HBNI, Jatni - 752050, Odisha, India
}

\begin{abstract}
Carbon $(\mathrm{C})$ doped hexagonal boron nitride(hBN) has been experimentally reported to be ferromagnetic at room temperature. Substitution by $\mathrm{C}$ in $\mathrm{hBN}$ has been also reported to form islands of graphene. In this work we derive a mechanistic understanding of ferromagnetism with graphene islands in hBN from first principles and mean-field Hubbard model. We find a general property, that in bipartite lattices where the sublattices differ in on-site energies, as in $\mathrm{hBN}$, the ordering between local magnetic moments can be substantial and predominantly anti-ferromagnetic(AFM) if they are embedded in the same sublattice, unless dominated by Mott like inter-sublattice spin separation due to strong localization. The dominant AFM order is rooted at spin resolved spatial separation of lone pairs of nitrogen $(\mathrm{N})$ and back transferred electrons on boron(B) due to Coulomb repulsion thus essentially implying a super-exchange pathway. Subsequently we propose a class of ferri-magnetically ordered inter-penetrating super-lattices of magnetic graphene islands in $\mathrm{hBN}$, which can be chosen to be a ferromagnetic semiconductor or a half-metal, and retain a net non-zero magnetic moment at room temperature.
\end{abstract}

\section{INTRODUCTION}

Possibility of ferromagnetism exclusively due to electrons in $2 p$ orbitals, particularly in the functionalized three coordinated bipartite networks [1, 2] of boron(B), carbon $(\mathrm{C})$ and nitrogen $(\mathrm{N})$, has opened up a new direction in pursuit of magnetic materials, which could be lighter and thinner than those made of traditional metals, besides having large spin relaxation time due to weaker spin-orbit coupling. Designing such materials which can also be magnetically as well as structurally stable at room temperature is thus a key objective in search for new paradigms of nano-fabrication. Magnetism in halffilled bipartite systems [3, 4, are primarily sourced at functionalizations due to structural, physical or chemical modification, which impact the two sublattices unequally and hinder $\pi$ conjugation thereby, leading to ferri-magnetic $(\mathrm{FeM})$ order between the sublattices then in general. Such functionalized bipartite systems would thus sustain a net non-zero magnetic moment, accompanied by finite density of states(DOS) for the minority spin at Fermi energy, leading to metallic phases allowing spin polarized transport. Many such scenarios of functionalization of graphene $(\mathrm{Gr})$ [5, 6], particularly in ribbons [7, 8] and finite segments with magnetic edges [9-12, have been widely proposed in the last decade or so.

However, presence of finite DOS near Fermi energy due to the inherent semi-metallic nature of graphene, and also due to spurious edge states, tend to undermine the effects of functionalizations, unless precisely cut into ribbons of systematic band gap, which poses its own experimental challenge. On the other hand hexagonal boron nitride(hBN) being a wide band gap due, do not interfere with the spin polarized DOS of graphene islands embedded in it, thus allowing greater scopes of their manipulation as per device requirements. Indeed, magnetic
Gr-hBN hybrids that have been widely proposed [1319] in last few years as a promising magnetic material made of non-metals, alongside several reports of success in robust synthesis 20 23. of such hybrid structures with arrays of Gr-islands embedded in hBN. Triangular Grislands with zigzag edges, which offer the largest magnetic moment 10 for a given island size, are expected to form stable motif $23-25$ in hBN. Neighboring magnetic Gr-islands embedded in hBN have been argued to prefer anti-ferromagnetic(AFM) 26, 27. ordering in proximity, although a less stable ferromagnetic(FM) phase has also been suggested at some specific proximities of islands. $\mathrm{C}$ doped $\mathrm{hBN}$ has been experimentally reported 28, to be ferromagnetic at room temperature in recent years. These results so far have been mostly anticipated mostly heuristically without any comprehensive closure on a microscopic mechanism. Thus the true nature of interaction between localized magnetic moments in $\mathrm{hBN}$ needed to be thorough established in order to systematically understand the observed results, and also to make rational proposals for new Gr-hBN hybrids with enhanced stability of the FM phase.

Thus the primary task that we set for us in this work, is to understand the exact mechanism of mediation of magnetic order by hBN between two Gr-islands with non-zero magnetic moment, followed by focus on stabilizing the FM phase, which led to systematic proposals for half-metal or ferromagnetic semiconductor with Gr-hBN hybrids, with their ferromagnetism sustainable at room temperature. Our study is based on analysis of spin polarized electronic structures calculated from first principles 29, as well as within the mean-field approximation of Hubbard model $4(\mathrm{MFH})$ in a tightbinding(TB) framework to establish our proposed phenomenological model. Effective strength of exchange interaction $(\mathrm{J})$ and transition temperatures are estimated within the Ising model of spin Hamiltonians 30 . Orbital 

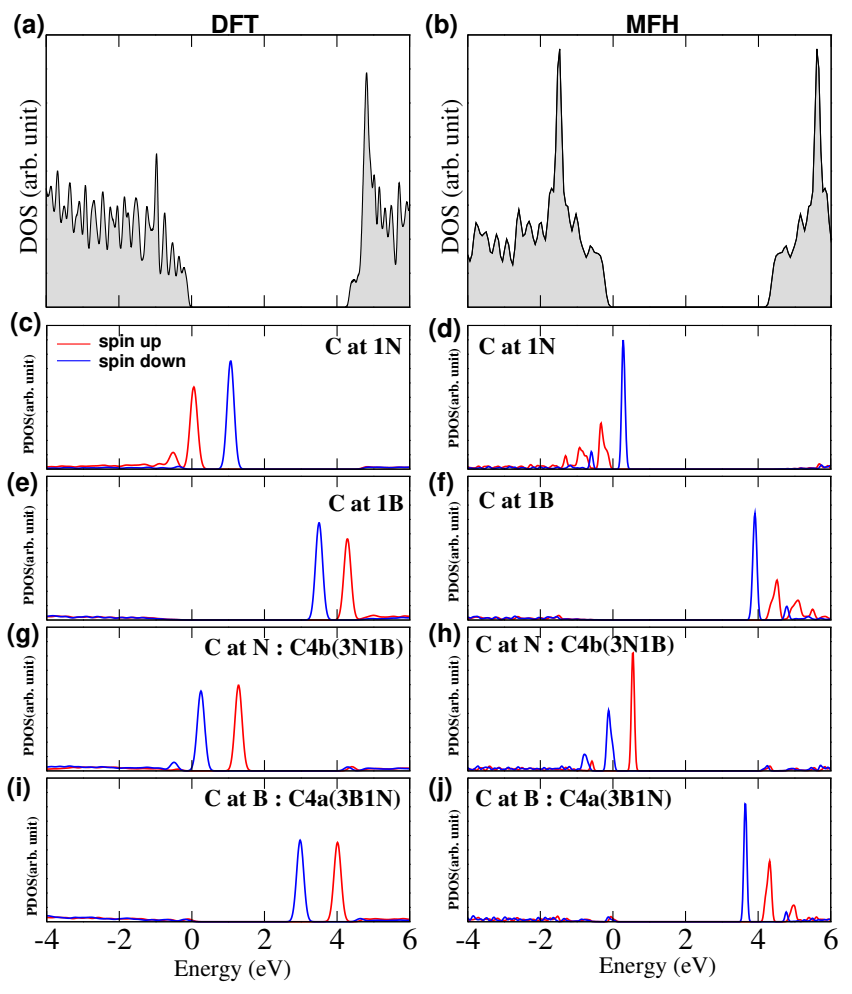

FIG. 1: Density of states(DOS) of pristine hBN from (a)DFT and (b)MFH model. Density of states projected on $2 p_{z}$ orbital of C-atom substituted at (c,d) single $\mathrm{N}$ site, (e,f) single B site, $(\mathrm{g}, \mathrm{h}) 3 \mathrm{~N}$ and $1 \mathrm{~B}$ sites, $(\mathrm{i}, \mathrm{j}) 3 \mathrm{~B}$ and $1 \mathrm{~N}$ sites, from spin polarized DFT(c,e,g,f) and MFH model(d,f,h,j).

resolved understanding of the underlying mechanisms have been developed based on Wannier functions 31, which are spatially localized linear combination of KohnSham eigen states, and are known to unambiguously divide the charge density into bonding and atomic orbitals.

\section{COMPUTATIONAL DETAILS}

Equilibrium configurations and spin polarized energetics are calculated within the framework of density functional theory (DFT) in plane wave basis 32, using ultrasoft pseudopotentials 33 and gradient corrected Perdew-Burke-Ernzerhof(PBE) exchange-correlation 34 functional. PBE results have been refined using the hybrid HSE 32, 35] approximation of exchange-correlation for better representation of exchange-interaction. However, given the computationally intensive nature of HSE calculations, only a representative variety of calculations have been refined using HSE. Total energies minimized using the BFGS 36 scheme, are converged with planewave cutoff over $800 \mathrm{eV}$, k-mesh equivalent to $30 \times 30$ for a hBN primitive cell and forces per atom less than $10^{-4}$ Rydberg/Bohr. Energetics of the ground states have been estimated starting from parallel(FM) and antiparallel(AFM) initial alignment of net magnetic moments of neighboring islands. Within each Gr-island we have considered anti-parallel alignment of spin at neighboring $\mathrm{C}$ sites. In all the calculations reported in this work FM or AFM ordering of the initial condition is retained in the ground state, unless they are non-magnetic. Therefore in cases where $\left(\mathrm{E}_{F M}-\mathrm{E}_{A F M}\right)$ is negligible, the corresponding ground states are either nonmagnetic, or are nearly degenerate. Spin polarized Wannier functions are constructed using subroutines 37 interfaced with Abinit 38 by maximally aligning the Kohn-Sham states with a template of one $2 p_{z}$ atomic orbital on each $\mathrm{C}$ atoms for one of the spins, two $2 p_{z}$ orbitals on each $\mathrm{N}$ atoms for the two spins, and two $\sigma$-bonding orbitals between all nearest neighbors for the two spins.

To rationalize a phenomenological model to explain the observed DFT results, electronic structure of $2 p_{z}$ electrons has been also calculated using MFH model, wherein

$$
H_{M F}=\sum_{i, \sigma} \varepsilon_{i} c_{i \sigma}^{\dagger} c_{i \sigma}+\sum_{<i, j>, \sigma} t_{i j} c_{i \sigma}^{\dagger} c_{j \sigma}+\sum_{i, \sigma} U_{i} n_{i \sigma}\left\langle n_{i \sigma^{\prime}}\right\rangle .
$$

Where $\varepsilon_{i}$ are the on-site energies $\left(\varepsilon_{B}, \varepsilon_{N}, \varepsilon_{C}\right), t_{i j}$ are nearest-neighbor hopping parameters $\left(t_{C C}, t_{B N}, t_{C N}\right.$, $\left.t_{C B}\right)$ and $U_{B}, U_{N}, U_{C}$ are the strength of on-site Coulomb repulsion. Since the valence shell of B, C, and $\mathrm{N}$ are of same principal quantum number $(n=2)$, we have set $U$ for all sites to that of $\mathrm{C}$ in graphene 2. We have also set the hopping parameters $t_{B N}, t_{C N}, t_{C B}$ to same as $t_{C C}$ of graphene [2]. Indeed various literatures 39 41] suggest a variation of $U$ and $t$ for different planer three coordinated self-assemblies with $\mathrm{B}, \mathrm{C}$ and $\mathrm{N}$ to be within $20 \%$ of those used for graphene on the average. Rather, as evident from Fig 1 $(a, b)$, we have matched the bulk band gap of hBN calculated from $\mathrm{MFH}$ to that from DFT to tune the on-site energies of $\mathrm{B}$ and $\mathrm{N}$, which are the only parameters to vary substantially from that of $\mathrm{C}$. After setting the on-site energy for $\mathrm{C}$ to zero, we find the on-site energies for $\mathrm{B}$ and $\mathrm{N}$ to be $3.1 \mathrm{eV}$ and $-3.1 \mathrm{eV}$ respectively. Comparison of density of states(PDOS) projected on $2 p_{z}$ orbitals[Fig]1(c-j)] imply satisfactory qualitative agreement between their DFT and MFH descriptions. The quality of agreement can be incrementally improved quantitatively further by marginally tuning $U$ and $t$ values, although inexhaustibly. However, our goal to demonstrate the validity of the mechanisms suggested by DFT results within the MFH model, has been satisfactorily accomplished with the MFH parameters used in this work.

Using first principles data we estimate the exchange coupling parameter $J$ and the corresponding transition temperature $\left(\mathrm{T}_{C}\right)$ by using the Ising model of honeycomb lattice considering only nearest-neighbor coupling, [30] as,

$$
T_{C}=\frac{2 J}{\ln (2+\sqrt{3})}
$$

where $-6 J=\left(E_{F M}-E_{A F M}\right)$, and $E_{F M}, E_{A F M}$ are the energies corresponding to FM and AFM configurations 
obtained from DFT. We thus refer $\left(E_{F M}-E_{A F M}\right)$ as the strength of magnetic ordering.

\section{RESULTS AND DISCUSSIONS}

Although there has been a steady rise in the number of computational[26, 27] as well as experimental[22, 23, 28. studies of graphene islands in hBN, attempts to understand the mechanism of mediation of magnetic order by hBN has been limited. From first principles calculations, magnetic islands in proximity in hBN have been argued to favor AFM ordering as a means to allow delocalization of spin densities 26] in conformity with Pauli exclusion principle. Similar AFM ordering has been reported in general for isolated $\mathrm{C}$ sites in $\mathrm{hBN}$ as well[18, 28, although, at some specific proximities and site coverages a less stable FM ordering has also been suggested [28] The strength of magnetic ordering has been shown to drastically reduce with increasing separation 26, 27. Energetically, FM and AFM ordering of magnetic islands in the ground state has been suggested to be metastable 27] and proposed to be determined by the $\mathrm{B}$ rich or $\mathrm{N}$ rich nature of the Gr-hBN interfaces around the islands, implying the magnetic ordering of Gr-islands to be a short-ranged. On the other hand, flat band based long-ranged mechanisms has been also anticipated to be responsible for FM ordering in $\mathrm{C}$ doped $\mathrm{hBN}[28$. FM ordering of free standing triangular Gr-islands through odd membered $\mathrm{C}$ chain has been shown possible due to the nearest neighbor AFM ordering of the bridge sites[11, 12] between Gr-islands, although such a mechanism is unlikely to be effective in case of triangular islands embedded in hBN. Attempts so far to explain the observed nature of magnetism in these Gr-hBN hybrid systems have thus remained largely speculative, and did not involve the possibility of hBN to take any active role, despite the spatial range of magnetic ordering being more than twice the magnitude of primitive lattice constant of $\mathrm{hBN}$. We probed this particular aspect in details and indeed found that interactions at the intervening $\mathrm{B}$ and $\mathrm{N}$ sites to play the central role in determining the nature of magnetic ordering of the Gr-islands.

As a source of magnetic moment in hBN, we started with isolated substitution by $\mathrm{C}$ denoted as C1(C1a:C@1B, C1b:C@1N), and have incrementally considered bigger islands made of three coordinated $\mathrm{C}$ : $\mathrm{C} 4 \mathrm{a}(\mathrm{C} 4 \mathrm{~b})$ covering three $\mathrm{B}(\mathrm{N})$ and one $\mathrm{N}(\mathrm{B})$ site, $\mathrm{C} 9$ covering six $\mathrm{B}$ and three $\mathrm{N}$ sites, and $\mathrm{C} 13$ covering seven $\mathrm{B}$ and six $\mathrm{N}$ sites, with magnetic moments $2 \mu_{B}, 3 \mu_{B}$ and $1 \mu_{B}$ respectively [3]. For bigger islands we have considered higher coverage of $\mathrm{B}$ site than that of $\mathrm{N}$ site, since substitution by $\mathrm{C}$ is known 42 to be energetically more favorable at $\mathrm{B}$ site than that at $\mathrm{N}$ site.

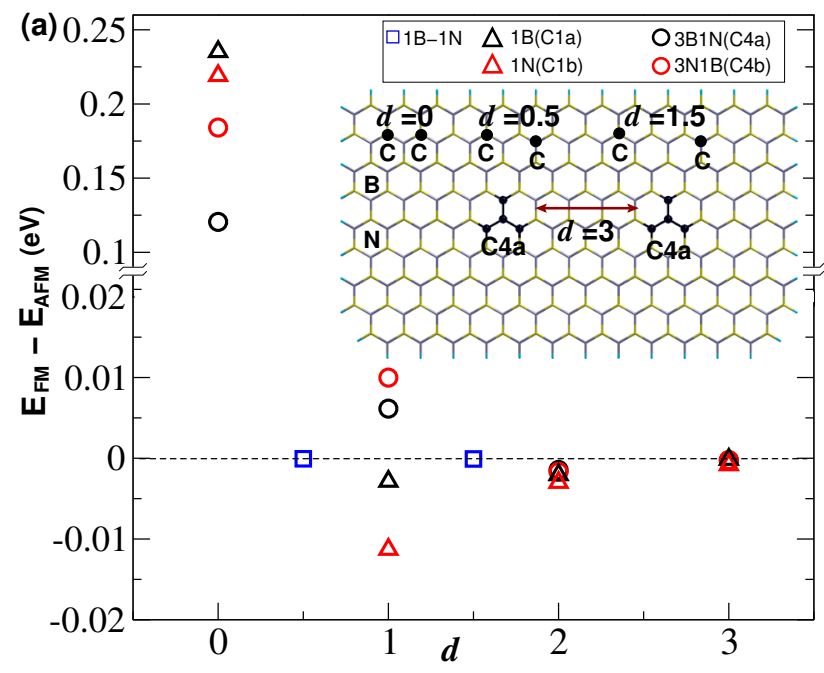

(b)

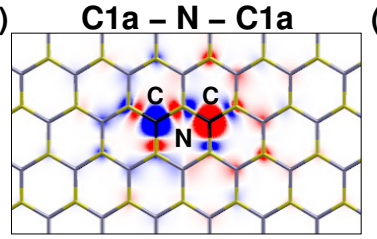

(c) $\quad C 1 b-B-C 1 b$

(d)
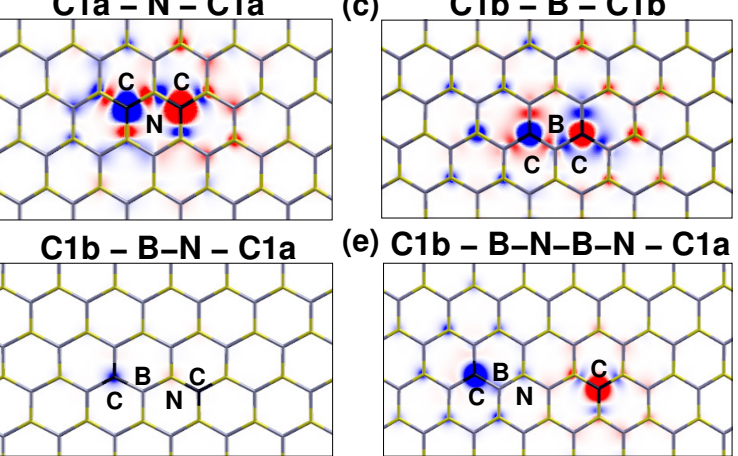

(e) $\mathrm{C} 1 \mathrm{~b}-\mathrm{B}-\mathrm{N}-\mathrm{B}-\mathrm{N}-\mathrm{C} 1 \mathrm{a}$

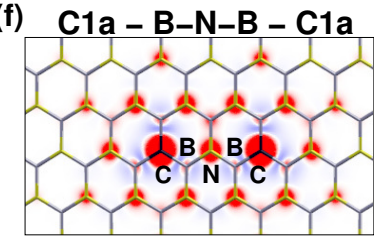

(h)
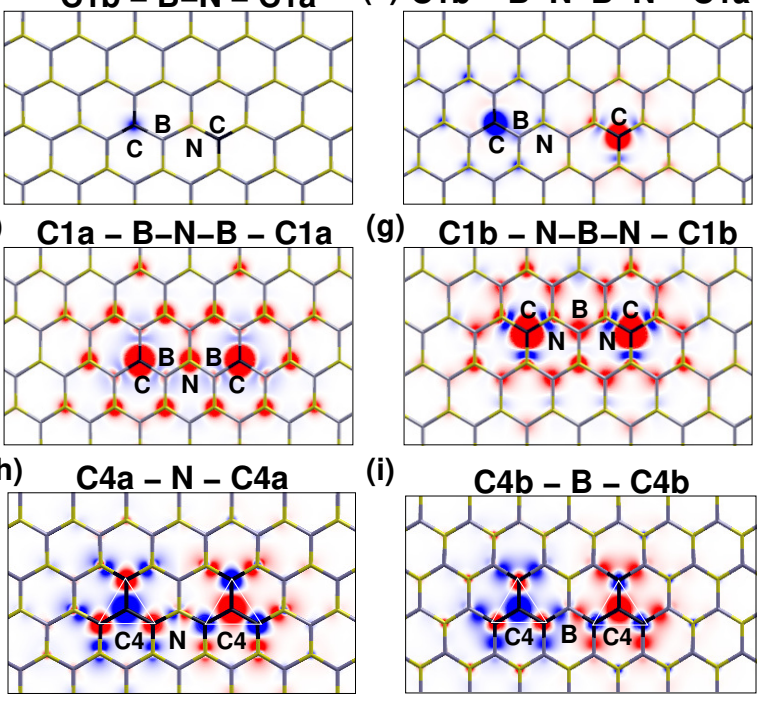

(g)

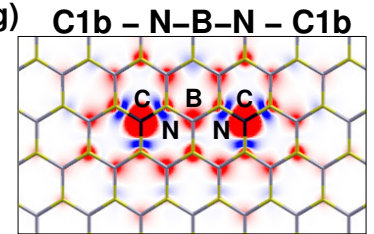

(i)

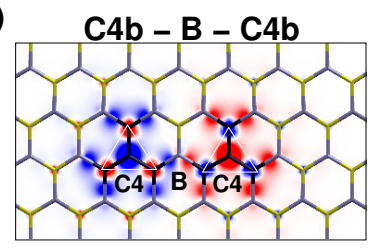

FIG. 2: (a) Energy difference $\left(\mathrm{E}_{F M}-\mathrm{E}_{A F M}\right)$ for two $\mathrm{C} 1$ sites(single substitution) and C4 islands for different island separation $d$ within a hydrogen passivated hBN segment. Spin densities with separation (b,c) $d=0$, (d) $d=0.5$, (e) $d=1.5$, $(\mathrm{f}, \mathrm{g}) d=1$ between two $\mathrm{C} 1$ sites, and $(\mathrm{h}, \mathrm{i})$ for separation $d=1$ between two $\mathrm{C} 4$ islands. All contour plots except (d) are in the range -0.001 to +0.001 . (d) is in the range -0.0001 to +0.0001 .

\section{A. Isolated pair of magnetic islands in $\mathrm{hBN}$ segment}

In Fig 2 (a) we plot the strength of magnetic ordering as a function of proximity $(d)$ of two graphene islands. As depicted in the inset of Fig.2(a), integer(fractional) values of $d$ imply location of the magnetic moments in the 
(a)

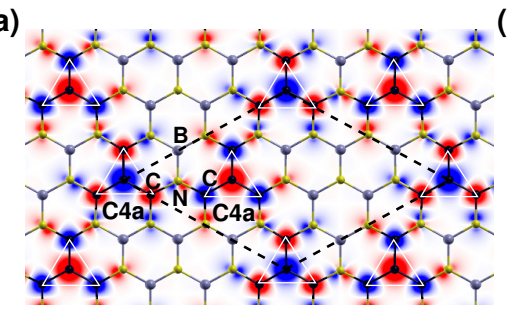

(d)

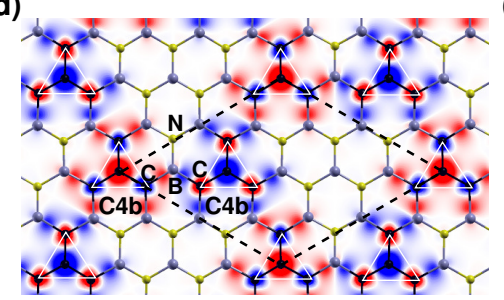

(b)

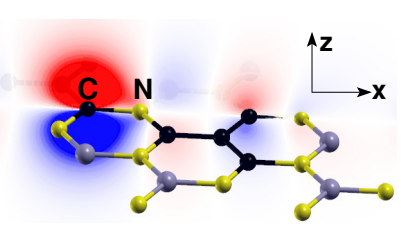

(e)

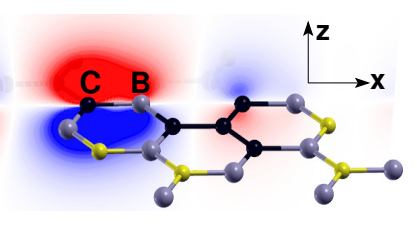

(c)

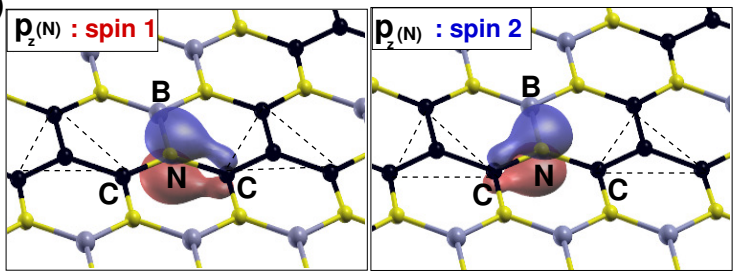

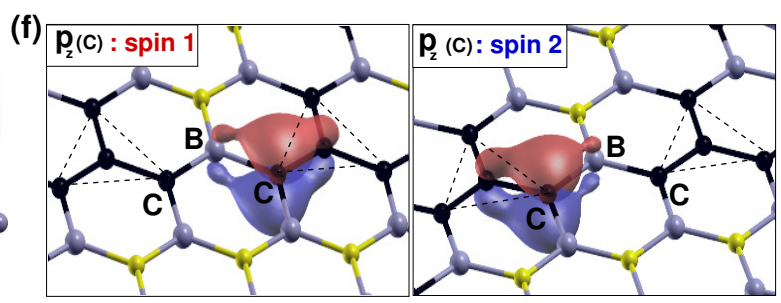

FIG. 3: Spin density of honeycomb super-lattice with (a) C4a(3B1N), (d) C4b(3N1B) with $d=0$. Planar projection of Wannier functions representing $2 p_{z}$ orbital of $\mathrm{C}$ along (b) C-N and (e) C-B bonds for honeycomb super-lattices made of C4a and C4b islands respectively. Spin-resolved Wannier function of $2 p_{z}$ orbital of (c) the bridging $\mathrm{N}$ atoms between $\mathrm{C} 4 \mathrm{a}(3 \mathrm{~B} 1 \mathrm{~N})$ islands and (f) the outer $\mathrm{C}$ atoms of a $\mathrm{C} 4 \mathrm{~b}(3 \mathrm{~N} 1 \mathrm{~B})$ island.

same(different) sublattice(s), leading to unequal(equal) number $\mathrm{B}$ and $\mathrm{N}$ sites between the local moments. As implied in Fig 2(a), for both $\mathrm{C} 1$ and $\mathrm{C} 4$ islands, our results suggest AFM ordering at close proximity $(d=0)$ irrespective of whether the islands are rich in B-site $(\mathrm{C} 1 \mathrm{a}, \mathrm{C} 4 \mathrm{a})$ or N-site(C1b, C4b). For $d=0$, spin densities of the AFM ordered ground state depicted in Fig, $2(\mathrm{~b}, \mathrm{c})$ and (h,i) for $\mathrm{C} 1$ and $\mathrm{C} 4$ respectively, both reveal spatial separation of electrons of opposite spins on the intermediate B and $\mathrm{N}$ sites, hinting at the possibility of a super-exchange 4] like mechanism leading to AFM ordering. At $d=1$, AFM order sustains between $\mathrm{C} 4$ islands but weak FM order emerges between $\mathrm{C} 1$ islands. Such switching of order from strong $\mathrm{AFM}$ at $d=0$ to weaker $\mathrm{FM}$ at $d=1$ for $\mathrm{C} 1$ hints at a competitive scenario of interactions. To rationalize the FM order, we note that in the absence of any mechanism to support AFM order, FM order between the $\mathrm{C}$ atoms at same sublattice would have been natural, owing to the on-site Coulomb repulsion driven Mott like inter-sublattice spin separation induced by the localized $2 p_{z}$ electrons of $\mathrm{C} 1$, as implied in Fig $2(\mathrm{f}, \mathrm{g})$. Such a mechanism would favor FM(AFM) ordering of local moments in the same(different) sub-lattice(s). The degree of localization of $2 p_{z}$ electrons is stronger in $\mathrm{C} 1$ than in Gr-islands due to the scope of delocalization of those electrons in the latter on account of $\pi$ conjugation. Therefore the Mott like spin separation would be induced more strongly by the $2 p_{z}$ orbital of $\mathrm{C} 1$, than by those in $\mathrm{C} 4$ or larger islands, which is likely the reason why at $d=1$ the FM order is observed only between $\mathrm{C} 1$ in the same sublattice, and not between C4. At $d=0$, such FM order is thus completely suppressed by the AFM order even for $\mathrm{C} 1$.

At $d=0.5$ and 1.5 both the mechanisms should have led to AFM order, instead, vanishing strength of magnetic ordering is observed for $d=0.5$ and 1.5. For $d=0.5$, the ground state has negligible magnetic ordering, while for $d=1.5$, the FM and AFM ordered states are effectively degenerate in energy, as apparent from the spin densities of the corresponding AFM ordered ground state plotted in Fig 2(d,e). Thus the AFM and FM order both appear to weaken if the connecting -B-N- pathways allow an equal number of $\mathrm{B}$ and $\mathrm{N}$ atoms. The likely reason for the weakening of the AFM is the asymmetric neighborhood of each of the intervening sites along the pathway connecting the two moments. For example, in -C-B-N$\mathrm{C}$ - pathway where the $\mathrm{C}$ atoms provide the local moments, neither B nor $\mathrm{N}$ has a symmetric neighborhood. Such denaturing of the super-exchange bridge is evident in Fig 2(e). Whereas, in case of a -C-B-N-B-C- (or -CN-B-N-C-) pathway, the $\mathrm{N}$ (or B) atom in the middle has symmetric neighborhood which forces formation of a super-exchange bridge on it, leading to AFM ordering between the $\mathrm{C}$ atoms. Weakening of the FM order with equal coverage of the two sublattices by the intervening sites can be understood by noting that in general Mott like separation of spins on equal footing among the two sublattices will be hindered due to their different on-site energies. On the other hand, with unequal coverage of the two sublattices by the intervening sites the separation of spins need not be on equal footing among the two sublattices as evident in Fig $2(\mathrm{f}, \mathrm{g})$, which conforms with their different on-site energies.

$\mathrm{C} 2$ islands due substitution at nearest neighboring sites, constitutes $\mathrm{C}=\mathrm{C}$ dimers with weak localization of $2 p_{z}$ electron on the $\mathrm{C}$ atom with $\mathrm{N}$ neighbors owing to the effective heteropolarity of the $\mathrm{C}=\mathrm{C} \pi$ bond. $\mathrm{C} 3$ islands will be magnetic due to unequal coverage of the two sublattices. C2 and C3 are expected to have similar magnetic ordering as $\mathrm{C} 4$, owing to weaker or similar localization of $2 p_{z}$ electrons compared to that in C4. Notably, at $d=2$, both $\mathrm{C} 1$ and $\mathrm{C} 4$ islands are weakly FM 
ordered, implying weakening of the AFM ordering. Thus the AFM ordering is stronger than FM ordering in close proximity but has a relatively shorter range than the FM ordering. Therefore, both the competing mechanisms are driven by the on-site Coulomb repulsion, but originate at different sites, which differ in occupancy, since AFM (FM) ordering can be generalized to originates at sites occupied by even(odd) number of electrons.

\section{B. Honeycomb lattice of magnetic islands}

In agreement with previous reports [26, we also find AFM ordering of sub-nanometer length-scale between neighboring Gr-islands in honeycomb lattice. Effective $\mathrm{J}$ estimated using HSE approximation of exchangecorrelation confirms that -B-N- zigzag connectivity between neighboring Gr-islands favor their AFM ordering, more than that due to -B-N- armchair connectivity [26]. This is in agreement with the result discussed in the previous section that magnetic ordering between two local moments in $\mathrm{hBN}$ would weakens if they are connected through -B-N- pathway having an equal number of $\mathrm{B}$ and $\mathrm{N}$ sites, as is the case with armchair connectivity. Strength of AFM order, as measured by J between the Gr-islands, is found more if mediated by B than by N, and reduces rapidly beyond $d=3$ for honeycomb lattice.

Spin densities in the vicinity of the intermediate $\mathrm{B}(\mathrm{N})$ atom between two $\mathrm{C} 4 \mathrm{~b}(\mathrm{C} 4 \mathrm{a})$ islands suggest[Fig $3(\mathrm{a}, \mathrm{d})]$ similar spatial separation of electrons of two spins on opposite sides of the $\mathrm{B}(\mathrm{N})$ atoms, as seen in Fig 2 (b-e). To trace the origin of this spin separation we looked at the spatially localized Wannier functions 37] representing the $2 p_{z}$ orbitals of $\mathrm{C}$ and $\mathrm{N}$. As evident in Fig 3 (c), the two unpaired $2 p_{z}$ orbitals of $\mathrm{N}$ with opposite spins extend spatially in opposite directions, resulting into back transfers of opposite spins to the $\mathrm{C}$ atoms on its two sides. Such a spatial splitting of lone pair is a hallmark of super-exchange pathway. Similarly on B atom between two C4b islands[Fig]3(f)], the back transfered electrons from the $2 p_{z}$ orbitals of the two neighboring $\mathrm{C}$ atoms are

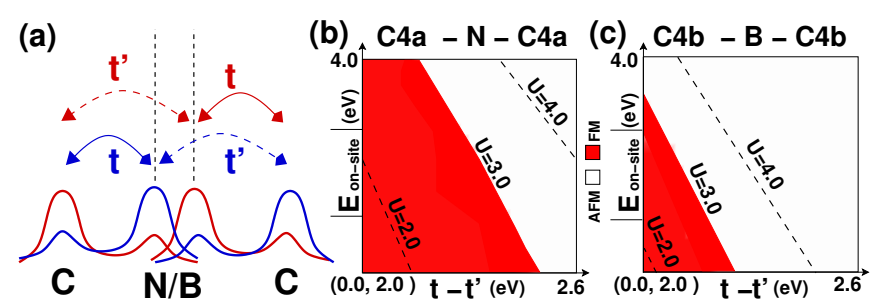

FIG. 4: (a): Schematic representation of spin dependent hopping mechanism. Phase diagram in terms of total magnetization of the ground state calculated as function of (t-t') and on-site energy $\mathrm{E}_{\text {on-site }}$ for a pair of (b): $\mathrm{C} 4 \mathrm{a}(3 \mathrm{~B} 1 \mathrm{~N})$ and $(\mathrm{c})$ : $\mathrm{C} 4 \mathrm{~b}(3 \mathrm{~N} 1 \mathrm{~B})$ islands with $d=0$ in a finite $\mathrm{hBN}$ segment at three different $\mathrm{U}$ values $(\mathrm{eV})$. For $\mathrm{U}=4.0 \mathrm{eV}$ and $\mathrm{U}=2.0 \mathrm{eV}$ the FM-AFM crossover is shown by black dotted line. (a)

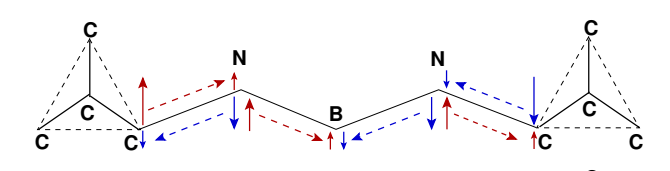

(b)

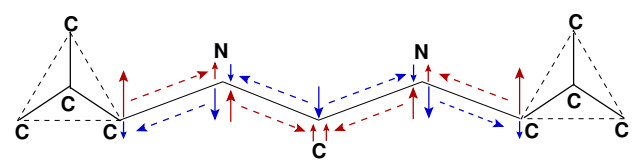

(c)

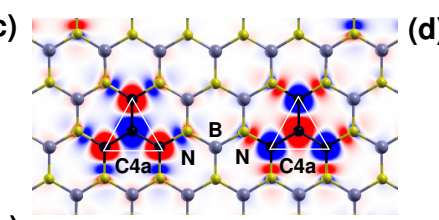

(e)

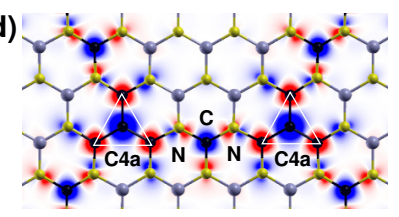

(f)

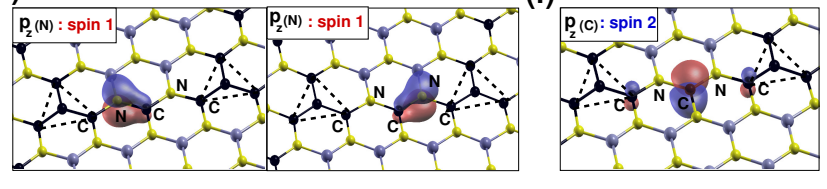

FIG. 5: Schematic model of (a): AFM ordered C4a(3B1N) islands. (b): FM ordered $\mathrm{C} 4 \mathrm{a}(3 \mathrm{~B} 1 \mathrm{~N})$ islands due to the extra local moment $(\mathrm{C})$ at $\mathrm{B}$ site between the $\mathrm{C} 4 \mathrm{a}$ islands. In $(\mathrm{a}, \mathrm{b})$ the dashed arrows represent back transfer of electrons to neighboring sites. Spin density plot of honeycomb superlattice of C4a islands with $d=1$ in (c): absence and (d): presence of $\mathrm{C}$ in the intermediate $\mathrm{B}$ sites. Wannier function representing the $2 p_{z}$ orbitals of (e): the two $\mathrm{N}$ atoms back transferring to the $\mathrm{C}$ in the middle, and (f): of the $\mathrm{C}$ atom itself, in the -N-C-N- zigzag pathway between $\mathrm{C} 4 a$ islands.

of opposite spins, implying spin separation about B as observed in the spin densities[Fig[3(a,d)]. Therefore, on $\mathrm{N}$ sites the orbitals offering the super-exchange bridge are fully occupied, while on the B site they are partially occupied by the back transferred electrons from neighboring $\mathrm{N}$ or $\mathrm{C}$ sites. Furthermore, Fig.3(b,e) imply that the back transfer from $\mathrm{C}$ to $\mathrm{B}$ is more than that to $\mathrm{N}$, which is consistent with the fact that the effective $\mathrm{J}$ is more for B mediated AFM than N mediated AFM.

\section{Mechanisms of mediation of magnetic ordering through hBN}

Super-exchange pathway leading to spin selective back transfer in effect indicates spin dependent hopping of electrons as a possible realization within a TB framework. We resort to MFH model to test the relevance of the super-exchange bridge represented by the spin dependent hopping, in determining the correct magnetic ordering of the ground state. Spatial separation of spins suggest symmetric opposite displacement of orbitals of opposite spins away from the host atom, as depicted in the schematic model[Fig 4(a)], which implies similar increase in the orbital energies (on-site term) for both the spins. Accordingly, we consider a pair of $\mathrm{C} 4$ islands in close proximity $(d=0)$ in the middle of a large hBN segment whose edges are sufficiently away from the $\mathrm{C} 4$ is- 
lands, and calculate AFM, FM and non-magnetic(NM) ground states as a function of spin-asymmetry of hopping $\Delta t=t_{\uparrow}-t_{\downarrow}$ applied to all the $(\mathrm{B} / \mathrm{N})-\mathrm{C}$ bonds around the $\mathrm{C} 4$ islands, and on-site term of the $(\mathrm{B} / \mathrm{N})$ atoms connected to the $\mathrm{C} 4$ islands, and look for the true magnetic ordering of the ground state by comparing total energies calculated in FM, AFM and NM conditions. We chose an isolated hBN segment in order to avoid the dependence of the magnetic ordering on the choice of periodic unit cell.

As evident from the TB-MFH based phase diagram shown in Fig $4(\mathrm{~b}, \mathrm{c})]$, the ground state with spin independent hopping and standard parameters, as discussed in sec III is FM ordered with total $4 \mu_{B}$ magnetic moment, which is in disagreement to DFT results. The AFM ordering of the ground state, emerges only beyond a threshold value of $\Delta t$, and the threshold $\Delta t$ itself decreases with decreasing $U$ as well as increase in on-site term. Spin dependent hopping is therefore crucial for the ground state to have AFM ordering of Gr-islands in agreement with DFT result. The fact that the $\Delta t$ threshold decreases with decreasing $U$, reiterates the role of $U$ at $\mathrm{B}$ and $\mathrm{N}$ sites in mediating the AFM order. In agreement with the fact the $\mathrm{B}$ mediated AFM is stronger than $\mathrm{N}$ mediated AFM, the onset of AFM order in the ground state indeed occurs at a lesser threshold for $\Delta t$ in case of $\mathrm{B}$ mediated AFM. Fig:5(a)], schematically summarizes the mechanism of propagation of AFM order through -B-Nzigzag pathway between Gr-islands, where the spatially separated spin polarized $2 p_{z}$ orbitals at $\mathrm{N}$ and $\mathrm{B}$ sites act as super-exchange bridges between local moments. The dashed arrows in Fig $5(a, b)$ indicates the direction
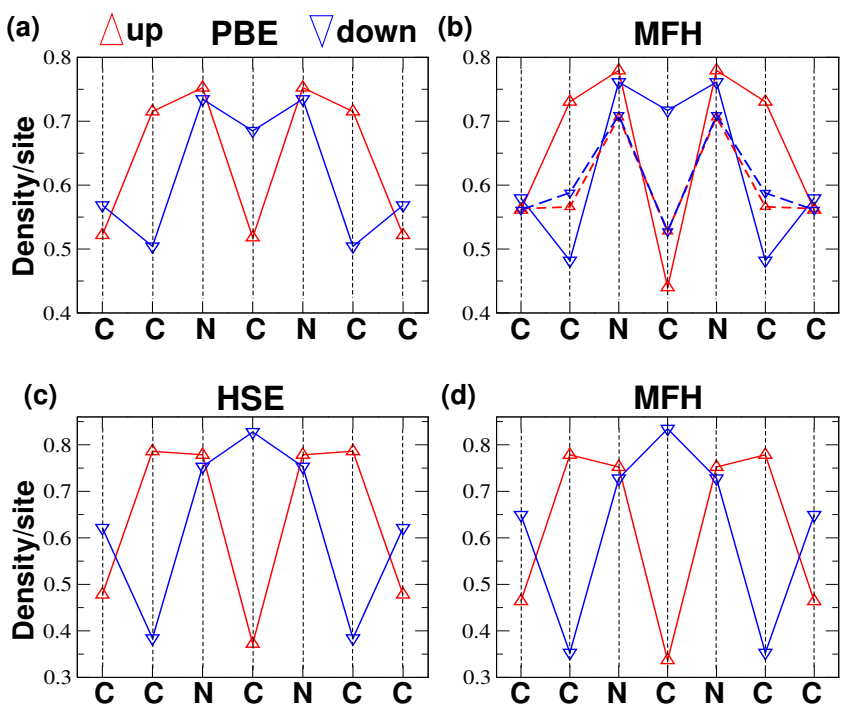

FIG. 6: Occupancy of $2 p_{z}$ orbitals of the two spins along zigzag path due to -N-C-N- connectivity between $\mathrm{C} 4 \mathrm{a}(3 \mathrm{~B} 1 \mathrm{~N})$ islands calculated using (a): PBE and (c)HSE, as well as from $\mathrm{MFH}$ with (b): $U=3.0 \mathrm{eV}$, and (d): $U=6.0 \mathrm{eV} . \Delta$ t set to 0.5 $\mathrm{eV}$ for (b) and (d). The occupation represented by the dotted lines in (b) corresponds to $\Delta \mathrm{t}=0.0 \mathrm{eV}$. of back transfer.

This mechanism thus implies a generic refinement of TB model for bipartite lattices if the sublattices have different on-site energies, and thereby are occupied differently. In fact, the mechanism points to a general property, that the magnetic ordering mediated by a bipartite system between two local magnetic moments would depend on the degree of asymmetry of the two constituent sublattices and the degree of localization the magnetic moment. With lesser asymmetry of the intervening sublattices, or stronger localization of the moment, Mott like spin separation will more likely to lead to FM order between magnetic moments in the same sublattice, as we see in graphene with local sublattice asymmetry due to functionalization. With increasing asymmetry of the intervening sublattices, or weaker localization of the moment, activation of super-exchange bridge at the sites of the sublattice with higher occupation is more likely to lead to AFM order of magnetic moments in the same sublattice. Since consolidation of both FM and AFM order requires the intervening pathway to be symmetric about the middle, magnetic ordering will be weaker in general between local moments at different sublattices.

\section{Ferromagnetic ordering}

The mechanism[Fig $[5(a)]$ also readily suggests that the AFM order can be switched to FM if an unpaired electron is present in the $-\mathrm{B}-\mathrm{N}$ - pathway connecting the two islands, as seen on the $\mathrm{C}$ site between the $\mathrm{N}$ sites in the -B-N- zigzag pathway between two $\mathrm{C} 4 \mathrm{a}$ islands in Fig 5(b). Such an unpaired electron can arise from a single substitution by $\mathrm{C}$, or from another magnetic $\mathrm{Gr}$ island located on or close to the intervening -B-N- pathway between the neighboring Gr-islands of the honeycomb lattice. Spin density in Fig.5(d), compared to that in Fig.5(c) without the intermediate $\mathrm{C}$ between the two $\mathrm{C} 4$ islands, indeed clearly confirms the anticipation of FM order of Gr-islands (C4a) connected by N-C-N- zigzag pathway. Facilitation of FM order due to spin separation of the $\mathrm{N}$ lone pairs is evident in Fig.5(e), where the two $\mathrm{N}$ atoms on two sides of $\mathrm{C}$ is seen to back transfer electrons of same spin (spin1) to $\mathrm{C}$ due to presence of a $2 p_{z}$ electron of spin2 at C[Fig [5(f)], which appears to open a halfmetallic bridge connecting the two Gr-islands. Note that the $2 p_{z}$ electron at the intermediate $\mathrm{C}[\mathrm{Fig} 5$ (f)] mixes with those of the $\mathrm{C} 4$ island through anti-bonding states.

Since even membered -B-N- pathway is expected to suppress mediation of magnetic order as argued above in Sec IIIA the generalization of the -N-C-N- pathway for strong FM ordering is $-(2 \mathrm{~d}+1 \mathrm{~B}$ and $\mathrm{N})-\mathrm{C}-\left(2 \mathrm{~d}^{\prime}+1 \mathrm{~B}\right.$ and $\mathrm{N})$-, where $\mathrm{d}$ and $\mathrm{d}^{\prime}$ are integers. These results suggest that in general an odd number of magnetic Gr-islands if located within nanometers of each other in $\mathrm{hBN}$, can be FeM ordered amounting to a net magnetic moment.

As evident from Fig.6(a,c) the FM ordering in the honeycomb lattice and the FeM ordering between honey- 
(a)

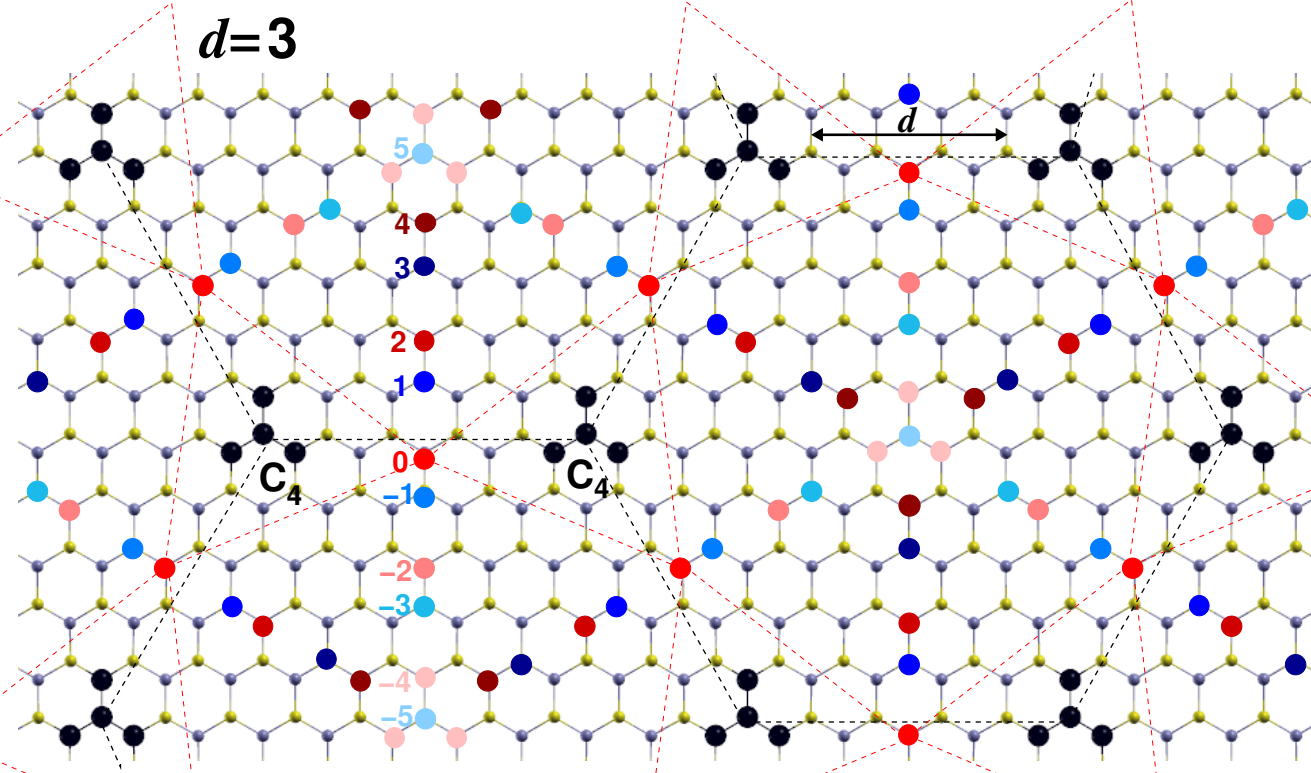

(b)

$d=1$

(c)

$d=2$

(d)

$d=3$

(e)

$d=4$
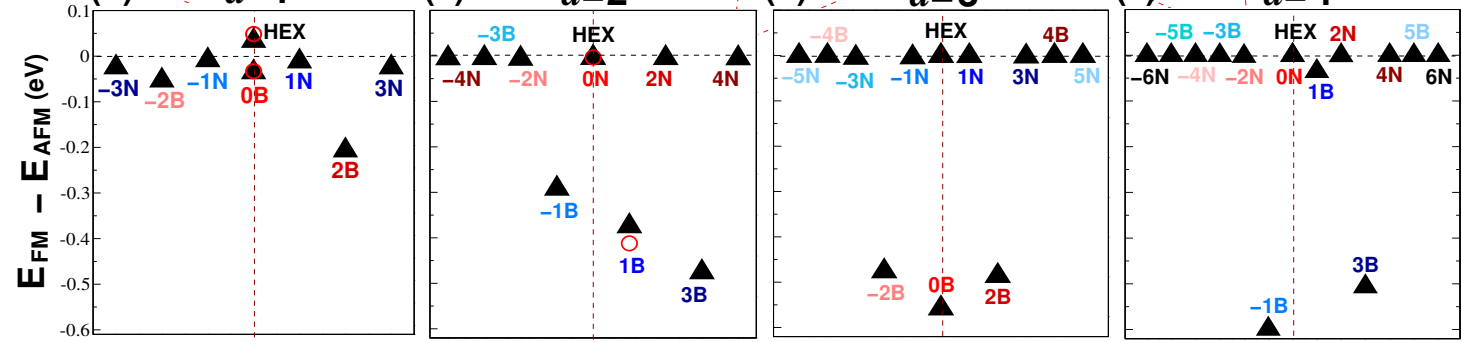

FIG. 7: (a):Representation of inter-penetrating C4a-X honeycomb-Kagome super-lattices for various intermediated substitution by $\mathrm{C}$ at $\mathrm{X}(\mathrm{B}$ or $\mathrm{N})$ sites for $d=3$. Energy difference $\left(E_{F M}-E_{A F M}\right)$ for different $\mathrm{X}$ different separations of $\mathrm{C} 4 \mathrm{a}$ islands,(b): $d=1,(\mathrm{c}): d=2,(\mathrm{~d}): d=3$ and $(\mathrm{e}): d=4$. Red circles in (b, c) represents corresponding energy difference with an additional layer of $\mathrm{hBN}$ at $\mathrm{A}-\mathrm{B}$ stacking beneath the hybrid layer as reported for hBN bilayer. $E_{F M}-E_{A F M}<0$ implies FM order in honeycomb super-lattice.

comb and kagome lattice enhances with HSE exchangecorrelation functional compared to that with PBE. Along the -N-C-N- pathway we have matched the PBE spin density with that due to $\mathrm{MFH}$ with $\mathrm{U}=3.0 \mathrm{eV}$ and $\Delta t=0.5$ $\mathrm{eV}$. Notably, with $\Delta t=0$ the FM ordering does not arise and spin separations are minimal[dashed line in Fig 6 (b)]. Since Hubbard Hamiltonian in effect evolves into a spin exchange Hamiltonian at high U, the HSE spin densities along the -N-C-N pathway [Fig 6(d)] matches with that due to $\mathrm{MFH}$ with $\mathrm{U}=6.0 \mathrm{eV}$ and $\Delta t=0.5 \mathrm{eV}$. This indispensability of $\Delta t$ being non-zero confirms the central role of spin dependent hopping in rationalizing the FM ordering of Gr-islands mediated by -N-C-N- pathway.

\section{Double lattices of Gr-islands in $h B N$}

As evident in Fig.7(a), the Gr-islands(C4), and the sites $(\mathrm{X})$ of the unpaired electron, which can be a $\mathrm{B}$ or a $\mathrm{N}$ site, describe a system of two inter-penetrating superlattices: one being the honeycomb super-lattice made by the $\mathrm{C} 4$ islands, while the other one is a twisted Kagome super-lattice made by $\mathrm{X}$ sites. The -B-C-B- or -N-C-Nmediated FM order of Gr-islands suggest a FeM order between the super-lattices. We now survey the variation in strength of the FM order in the hexagonal super-lattice as a function of location of the $\mathrm{X}$ site, which determines the twist of the Kagome lattice, as depicted in Fig 7(a). The energy difference $\mathrm{E}_{F M}-\mathrm{E}_{A F M}$ plotted in Fig.7(be) for C4a-X honeycomb-Kagome (H-K) super-lattices, suggest a systematic emergence of strong FM ordering in the hexagonal super-lattice, if the $\mathrm{X}$ site allow an odd membered -B-N- pathway connecting the Gr-islands. As evident in Fig.7(b-e), this is possible only with $\mathrm{X}: \mathrm{B}(\mathrm{N})$ for the honeycomb lattice made of $\mathrm{C} 4 \mathrm{a}(\mathrm{b})$. That even membered -B-N- pathway suppress propagation of magnetic order, is reiterated by the observation that strong FM order between C4a islands occurs only for X:B, since such $\mathrm{X}$ connects to nearest $\mathrm{C} 4 \mathrm{a}$ island through odd membered -B-N- pathway. X should also be typically within $7 \AA$ from the shortest -B-N- zigzag pathway connecting two neighboring $\mathrm{C} 4 \mathrm{a}$ islands. Similar results exist for C4b islands as well as bigger $\mathrm{C} 9(6 \mathrm{~B} 3 \mathrm{~N})$ islands in the hexagonal super-lattice. In fact, these results are valid for 

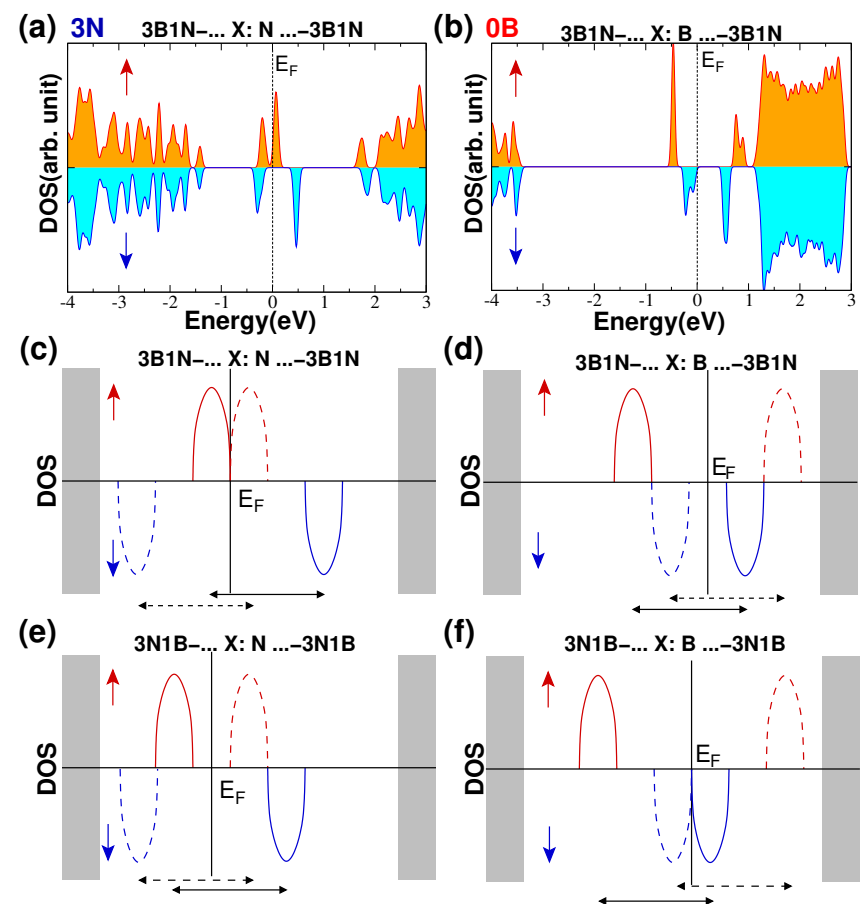

FIG. 8: Spin-polarized density of states(DOS) for (a): $\mathrm{X}=3 \mathrm{~N}$ and (b): $\mathrm{X}=0 \mathrm{~B}$ as described in Fig $7(\mathrm{a}, \mathrm{d})$ for $d=3$. (c-f): Schematic presentation of DOS to understand its evolution of half-metallic and FM-Sc phases with different choices of C4 islands (C4a or $\mathrm{C} 4 \mathrm{~b})$ and $\mathrm{X}(\mathrm{N}$ or $\mathrm{B})$.

a general Cm-Cn H-K super-lattices, where $\mathrm{Cm}$ and $\mathrm{Cn}$ are two magnetic Gr-islands constituting the two lattices, and can be chosen to be same or dissimilar. The corresponding $T_{C}$ estimated using eqn 2 indicates existence of FM order at room temperature. Fig. 7 (b-e) also suggests a $d$ dependence of strength of the FM order, which can be understood in terms of the competition between the inherent AFM order of the Gr-islands in the honeycomb lattice and the FM order induced by the Kagome lattice of $\mathrm{X}$ sites. With increasing $d$ the strength of the AFM order reduces, leading to a peak of the FM order, which reduces with further increase of $d$ beyond 4, implying in effect a length-scale of nanometer. The impact of having an additional layer of $\mathrm{hBN}$ in A-B stacking geometry at a depth of 3.15Aas known for hBN bilayer [43, is indeed minimal $[\mathrm{Fig}] 7(\mathrm{~b}, \mathrm{c})]$ in terms of retention of ferromagnetism.

\section{FM ordered phases}

FM ordering in the honeycomb super-lattice leads to FM-semiconducting(FM-Sc) [Fig, 8(b)] to half-metallic [Fig, 8(a)] phases depending on $\mathrm{X}$ being a $\mathrm{B}$ site or an $\mathrm{N}$ site in C4a-X H-K super-lattices. Emergence of FMSc or half-metallic phases can be understood in terms of relative shifts in energies of (1): the $2 p_{z}$ orbitals of the $\mathrm{C}$ atoms at the edges of the Gr-islands, and (2): those of the $\mathrm{C}$ atoms at $\mathrm{X}$ site. DOS of the two sets of orbitals are represented by solid and dashed lines respectively in the schematic DOS shown in Fig.8 (c,d,e,f), drawn on the basis of orbital projected density of states of different configurations shown in Fig,7. The relative shift in energies of these two sets of orbitals, which are mechanistically of opposite spins, can be understood in terms of the difference in their localization owing to their different size $(\mathrm{C} 4$ and $\mathrm{C} 1)$ and distribution(honeycomb and Kagome). Fig $8(\mathrm{e}, \mathrm{f})$ and Fig $8(\mathrm{c}, \mathrm{d})$ suggests that the properties of the $\mathrm{C} 4 \mathrm{~b}-\mathrm{X}: \mathrm{B}(\mathrm{N})$ and $\mathrm{C} 4 \mathrm{a}-\mathrm{X}: \mathrm{N}(\mathrm{B}) \mathrm{H}-$ $\mathrm{K}$ super-lattices will be similar. With same $\mathrm{C} 4 \mathrm{a}$ islands in the honeycomb lattice, Fig 8 (c) and Fig 8 (d) explains emergence of half-metallic and FM-Sc phases due to X:N and X:B respectively, in agreement with DFT results shown in Fig,8(a) and Fig 8(b). Fig.8(c) and Fig 8 (f) suggests emergence of half-metallic windows of opposite spins at Fermi energies due to C4a-X:N and C4b-X:B H$\mathrm{K}$ super-lattices respectively, which is consistent with the DOS plotted in Fig.9(c) and (a) respectively, where the Kagome lattices $(\mathrm{X})$ are made of $\mathrm{N}$ site rich $\mathrm{C} 4 \mathrm{~b}$ and $\mathrm{B}$ site rich $\mathrm{C} 4 \mathrm{a}$ islands instead of a single $\mathrm{C}$ at $\mathrm{N}$ or $\mathrm{B}$ site respectively. The robustness of the half-metallic phases with $\mathrm{X}: \mathrm{C} 4 \mathrm{a} / \mathrm{C} 4 \mathrm{~b}$ suggests that the key to make the half metallic window broader is to increase delocalization of the spin polarized electrons at the Kagome lattice.

Fig $8($ d) and (e) suggests FM-Sc phases in C4a-X:B and $\mathrm{C} 4 \mathrm{~b}-\mathrm{X}: \mathrm{N}$ H-K super-lattices, which are consistent with the FM-Sc phases shown in Fig 9 (b) and (d) for C9-C4a and C13-C4a H-K super-lattices. Notably, the narrowing of FM-Sc band-gap from Fig 9 (b) to (d) can possibly be attributed to the increased delocalization of electrons in C13 than in C9 due to enhanced $\pi$ conjugation in the former. The coinciding DOS of the two spins in Fig 9(b) near Fermi energy can be understood by noting that the degree of localization of the $2 p_{z}$ electrons are similar in C4 and C9 islands. These results thus lead to a simple thumb-rule that a half-metallic(FMSc) phase is expected is the Gr-islands in the honeycomb and the Kagome lattices are rich in substitution at dissimilar(similar) sites out of $\mathrm{B}$ or $\mathrm{N}$, and increased delocalization of $2 p_{z}$ electrons in the Gr-islands constituting the honeycomb(Kagome) lattice would lead to wider(narrower) half-metallic(FM-Sc) window(gap).

\section{CONCLUSION}

To conclude, we show that Coulomb repulsion driven spatial separation of lone pairs and back transferred electrons of opposite spins on $\mathrm{N}$ and $\mathrm{B}$ atoms, implying in effect a super-exchange pathway, to be the primary mechanism for the anti-ferromagnetic order mediated by -B$\mathrm{N}$ - pathway between neighboring magnetic Gr-islands in hexagonal boron nitride. A weaker ferromagnetic order between local moments in the same sublattice also exists due to the generic Mott like inter-sublattice spin separation driven by on-site Coulomb repulsion, but sup- 


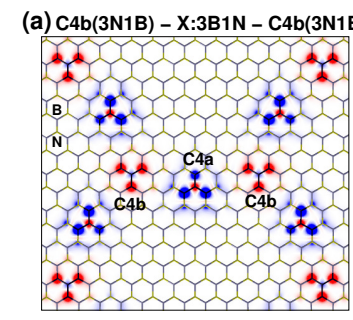

(b) $C 9(6 \mathrm{~B} 3 \mathrm{~N})-\mathrm{X}: 3 \mathrm{~B} 1 \mathrm{~N}-\mathrm{C}(6 \mathrm{~B} 3 \mathrm{~N})$

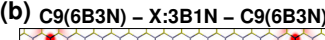

(b) $\mathrm{C} 9(6 \mathrm{~B} 3 \mathrm{~N})-\mathrm{X}: 3 \mathrm{~B} 1 \mathrm{~N}-\mathrm{C}(6 \mathrm{~B} 3 \mathrm{~N}$

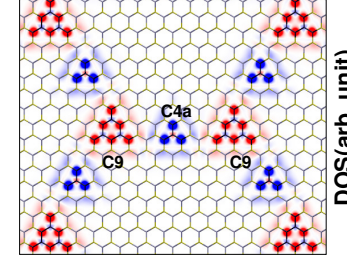

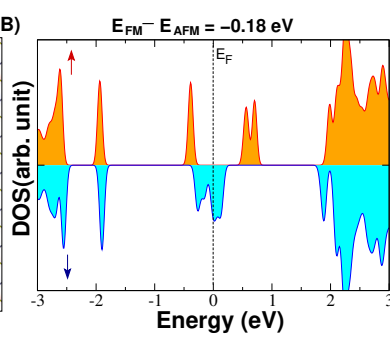

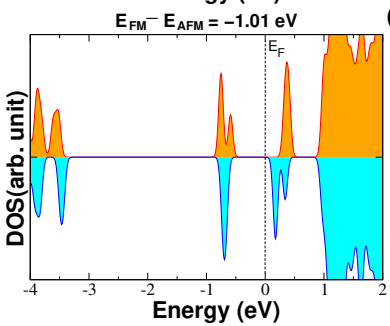

(c) $\mathrm{C}(6 \mathrm{~B} 3 \mathrm{~N})-\mathrm{X}: 3 \mathrm{~N} 1 \mathrm{~B}-\mathrm{C9}(6 \mathrm{~B} 3 \mathrm{~N})$

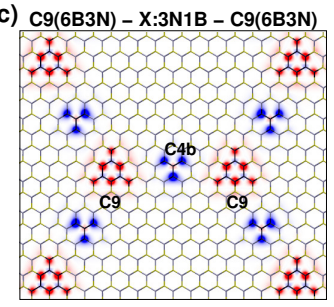

(d) $C 13(7 \mathrm{~B} 6 \mathrm{~N})-\mathrm{X}: 3 \mathrm{~B} 1 \mathrm{~N}-\mathrm{C13}(7 \mathrm{~B} 6 \mathrm{~N})$

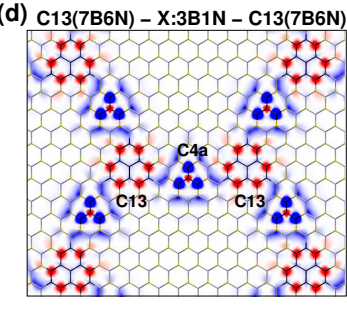

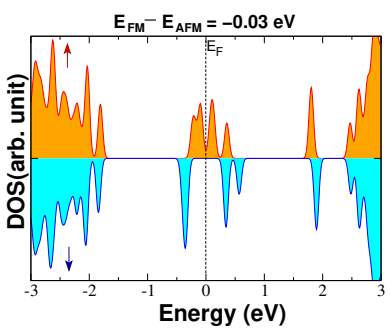

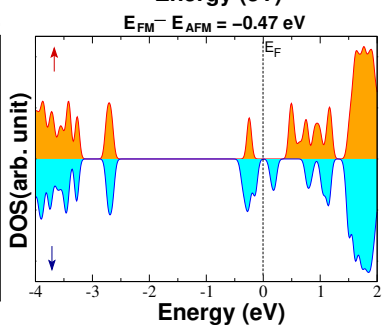

FIG. 9: Spin density and DOS for (a): C4b-C4a, (b): C9-C4a, (c) C9-C4b, (d) C13-C4a honeycomb-Kagome super-lattices, where C9:6B3N,C4a:3B1N, C4b:3N1B and C13:7B6N.

pressed by the super-exchange mechanism effective between graphene-islands, owing to weaker localization of $2 p_{z}$ orbitals with increasing island size. The mediated magnetic order becomes ferromagnetic if an odd number of unpaired spin interjects the -B-N- pathway, implying in general a finite net magnetic magnetic moment due to odd number of magnetic islands in proximity. An inter-penetrating system of honeycomb and twisted Kagome super-lattices of magnetic graphene-islands in $\mathrm{hBN}$ is proposed, wherein, the two ferri-magnetically ordered super-lattices should retain a net non-zero magnetic moment at room temperature, besides constituting a ferromagnetic semiconductor or a half-metal depending on the nature of Gr-islands in the two lattices.

\section{A. Acknowledgement}

This work has been performed in a high performance computing facility funded by Nanomission(SR/NM/NS1026/2011) of the Dept. of Sci. and Tech. of the Govt. of India. RM acknowledges financial support from the Dept. of Atomic Energy of the Govt. of India.
[1] O Volnianska and P Boguslawski, Journal of Physics: Condensed Matter, 22(7), (2010).

[2] O. V. Yazyev, Reports on Progress in Physics, 73(5), (2010).

[3] E. H. Lieb, Phys. Rev. Lett., 62,1201, (1989).

[4] P. Fazekas, Series in Modern Condensed Matter Physics, Vol.5 World Scientific Publishing Co. Pte. Ltd. (1999)

[5] Yan Wang et al., Nano Lett., 9(1), 220-224, (2009).

[6] J. Zhou, Q. Wang, Q. Sun, X. S. Chen, Y. Kawazoe and P. Jena, Nano Lett., 9 (11), 3867-3870, (2009).

[7] Young-Woo Son, Marvin L. Cohen \& Steven G. Louie, Nature, 444, 347-349, (2006).

[8] Sudipta Dutta, Arun K. Manna, and Swapan K. Pati, Phys. Rev. Lett., 102, 096601, (2009).

[9] W Sheng, Z. Y. Ning, Z. Q. Yang and H. Guo, Nanotechnology, 21(38), (2010).

[10] Wei L. Wang, Sheng Meng and Efthimios Kaxiras, Nano Lett., 8(1), 241-245, (2008).

[11] Xiaowei Li and Qian Wang, Phys. Chem. Chem. Phys., 14, 2065-2069, (2012).

[12] Jian Zhou, Qian Wang, Qiang Sun and Puru Jena, Phys. Rev. B, 84, 081402(R), (2011).

[13] Hongzhe Pan et al., New J. Phys., 18, 093021, (2016).
[14] Jin Choi, Yong-Hyun Kim, K. J. Chang, and David Tománek, Phys. Rev. B, 67, 125421, (2003).

[15] Somnath Bhowmick, Abhishek K. Singh, and Boris I. Yakobson, J. Phys. Chem. C, 115(20), 9889-9893, (2011).

[16] Yuling Liu, Xiaojun Wu, Yu Zhao, Xiao Cheng Zeng, and Jinlong Yang, J. Phys. Chem. C, 115(19), 9442-9450, (2011).

[17] J. M. Pruneda, Phys. Rev. B, 81, 161409(R), (2010).

[18] I. Choudhuri, B. Pathak, Chem Phys Chem, 19, 153-161, (2018).

[19] Tisita Das, Soubhik Chakrabarty, Y. Kawazoe, and G. P. Das, AIP Advances, 8, 065111, (2018).

[20] Yabo Gao et al., Nano Lett., 13(7), 3439-3443, (2013).

[21] Mengxi Liu et al., Nano Lett., 14(11), 6342-6347, (2014).

[22] Xianlong Wei, Ming-Sheng Wang, Yoshio Bando, and Dmitri Golberg, ACS Nano, 5(4), 2916-2922, (2011).

[23] Zheng Liu et al., Nature Nanotechnology, 8, 119-124, (2013).

[24] Yuanyue Liu, Somnath Bhowmick, and Boris I. Yakobson, Nano Lett., 11, 3113-3116, (2011).

[25] Jiong Lu, Lídia C. Gomes, Ricardo W. Nunes, A. H. Castro Neto and Kian Ping Loh, Nano Lett., 14(9), 51335139, (2014). 
[26] Ashwin Ramasubramaniam, Doron Naveh, Phys. Rev. B, 84, 075405, (2011).

[27] Mina Maruyama and Susumu Okada, J. Phys. Chem. C, 120, 1293-1302, (2016).

[28] Chong Zhao et al., Adv. Funct. Mater., 24, 5985-5992, (2014).

[29] W. Kohn, L. J. Sham, Physical Review., 140(4A), A1133-A1138, (1965).

[30] J. Baxter, Exactly Solved Models in Statistical Mechanics (Academic Press, London, (1982).

[31] G. Wannier, Phys. Rev., 52, 191, (1937).

[32] P. Giannozzi, S. Baroni, N. Bonini, M. Calandra, R. Car, C. Cavazzoni, D. Ceresoli, G. L. Chiarotti, M. Cococcioni, I. Dabo, et al., J. Phys.: Cond. Mat., 21, 395502(120), (2009).

[33] D. Vanderbilt, Phys. Rev. B, 41(R), 7892-7895, (1990).

[34] J. P. Perdew, K. Burke, M. Ernzerhof, Phys. Rev. Lett., 77, 3865-3868, (1996).

[35] Jochen Heyd, Gustavo E. Scuseria, and Matthias Ernzer- hof, The Journal of Chemical Physics, 118, 8207, (2003).

[36] R. Fletcher, Practical Methods of Optimization; Wiley: New York; (1987).

[37] J. Bhattacharjee, U. V. Waghmare, Phys. Rev. B(R), 73, 121102(1-4), (2006).

[38] X. Gonze, et. al, Comput. Mater. Sci., 25, 478-492. (2002).

[39] Fawei Zheng, Ken-ichi Sasaki, Riichiro Saito, Wenhui Duan, and Bing-Lin Gu, J. Phys. Soc. Jpn., 78, 074713, (2009).

[40] Tomoaki Kaneko, Kikuo Harigaya, and Hiroshi Imamura, J. Phys. Soc. Jpn., 82, 083710, (2013).

[41] Tomoaki Kaneko and Kikuo Harigaya, J. Phys. Soc. Jpn., 82, 044708, (2013).

[42] Natalia Berseneva, Arkady V. Krasheninnikov, and Risto M. Nieminen, Phys. Rev. Lett., 107, 035501, (2011).

[43] Yoshitaka Fujimoto and Susumu Saito, Phys. Rev. B, 94, 245427, (2016). 